\title{
Near-infrared oligonucleotide duplex sensors for imaging rapidly activated transcription factors in vitro and in situ
}

Alexei Bogdanov, Valeriy Metelev, Toloo Taghian, Ilya Solovyev, Anand T. Kumar, et al.

Alexei A. Bogdanov Jr., Valeriy G. Metelev, Toloo Taghian, Ilya Solovyev, Anand T. N. Kumar, Surong Zhang, Alexander Savitsky, "Near-infrared oligonucleotide duplex sensors for imaging rapidly activated transcription factors in vitro and in situ," Proc. SPIE 10877, Dynamics and Fluctuations in Biomedical Photonics XVI, 1087702 (1 March 2019); doi: 10.1117/12.2511124

SPIE. Event: SPIE BiOS, 2019, San Francisco, California, United States 


\title{
Near-infrared oligonucleotide duplex sensors for imaging rapidly activated transcription factors in vitro and in situ
}

\author{
Alexei A. Bogdanov Jr. ${ }^{1,3}$, Valeriy G. Metelev ${ }^{1,2}$, Toloo Taghian ${ }^{1}$, llya Solovyev ${ }^{2,3}$, Anand \\ T.N.Kumar ${ }^{4}$, Surong Zhang ${ }^{1}$, Alexander Savitsky ${ }^{2,3}$ \\ ${ }^{1}$ University of Massachusetts Medical School, Radiology, Worcester, Massachusetts, \\ United States of America \\ ${ }^{2}$ M.V.Lomonosov Moscow State University, Department of Chemistry, Moscow, Russian \\ Federation \\ ${ }^{3}$ A.N.Bach Institute of Biochemistry, Federal Research Centre "Fundamentals of \\ Biotechnology", Russian Academy of Sciences, Moscow, Russian Federation \\ ${ }^{4}$ A. Martinos Center for Biomedical Imaging, Massachusetts General Hospital, \\ Charlestown MA
}

\begin{abstract}
Pleiotropic and evolutionally conserved components of transcription nuclear factor - NF-кB play key roles in progression of various diseases by regulating expression of antiapoptotic and cytokine responsive genes [1] [2]. We previously demonstrated that rapidly activating transcription factors (TF) can be detected by using sequence-specific self-quenched reporter probes (oligonucleotide-molecular sensors (ODN-MS), which ideally remain "silent" in the absence of activated TF but emit photons upon specific binding to them [3-5]. Recently we were investigating sensor-based optical imaging of early inflammation in the endocrine pancreas using type 1 diabetes (T1D) model because NF-kB activation is essential for determining the fate of pancreatic $\beta$-cells and hence the progression of T1D. Using an immunocompetent SKH1 mouse model of early stage T1D we showed that NF-kB activation was induced by low-dose streptozotocin (LD-STZ). ODN-MS probes that carried near-infrared (NIR) fluorophores formed a complex with NF-kB subunits in in vitro assays and in situ after LD-STZ treatment. Imaging studies of pancreas (sections and isolated islets) were corroborated with electrophoresis mobility shift assays (EMSA). A higher specific NIR fluorescence intensity in nuclei and cytoplasm of islets from LD-STZ treated groups compared to non-treated control animals was observed. Our results demonstrate that: 1) the use of ODN-MS probes in non-fixed islets and tissue sections may be used for distinguishing differences in inflammatory pathway activation in animal models of early stage diabetes; 2) early, non-invasive detection of $\mathrm{NF}-\mathrm{kB}$ in pancreatic islets may serve as a potential strategy for imaging of early T1D-mediated sustained proinflammatory changes in the endocrine pancreas.
\end{abstract}

Keywords: oligonucleotide, duplex, transcription factor, near-infrared, fluorescence

\section{INTRODUCTION}

\subsection{The significance of transcription activation imaging}

Molecular dissection gene expression patterns in various pathologies that are driven by inflammation (such as cancer, atherosclerosis, diabetes etc.) has revealed hundreds of potential targets for therapy. Those targets include the components of normal as well as abnormal transcription machinery. Proteins involved in regulation of transcription of pro-inflammatory cytokines attain high priority due to the convergence of multiple signal transduction pathways at the transcription level. Novel molecular therapies directed to target gene transcripts, including RNA interference technology have potential advantages over traditional therapies due to a precision of their interference with target gene expression. Consequently, small molecule inhibitors, DNA-binding oligoamines, protein-binding oligonucleotide decoys as well as small hairpin RNAs and their combinations are being currently developed for therapy. While rapid progress in molecular genetics and medicinal chemistry delivers new «attenuators» of gene expression, there is a critical need in developing

Dynamics and Fluctuations in Biomedical Photonics XVI, edited by Valery V. Tuchin, Martin J. Leahy, Ruikang K. Wang Proc. of SPIE Vol. 10877, 1087702 · C 2019 SPIE · CCC code: 1605-7422/19/\$18 · doi: 10.1117/12.2511124 
technologies that enable early and non-invasive assessment of response to these therapies. In particular, enabling imaging technologies that report directly on gene transcription in living cells are critically important for phenotyping and staging of disease, as well as for evaluating new therapies. For two decades nearinfrared (NIR) fluorescent probes were successfully used for optical imaging of elevated proteolytic activity in situ and in vivo, including models of cancer and inflammation [10, 11]. To provide the insight into upstream/general regulatory elements that cause sustained inflammation and control pro- and antiinflammatory signaling we focused on designing ODN-MS for sensing interactions with transcription factors [12]. Rapidly activated, ubiquitous transcription factors (TF), e.g. members of the NF- $\kappa B$ family are intricately involved in inflammatory signaling [3-5]. Our recent progress in developing STAT3 transcription factor imaging probes $[8,13]$ anticipated the discovery of convergence between STAT3 and NF- $\mathrm{KB}$ pathways

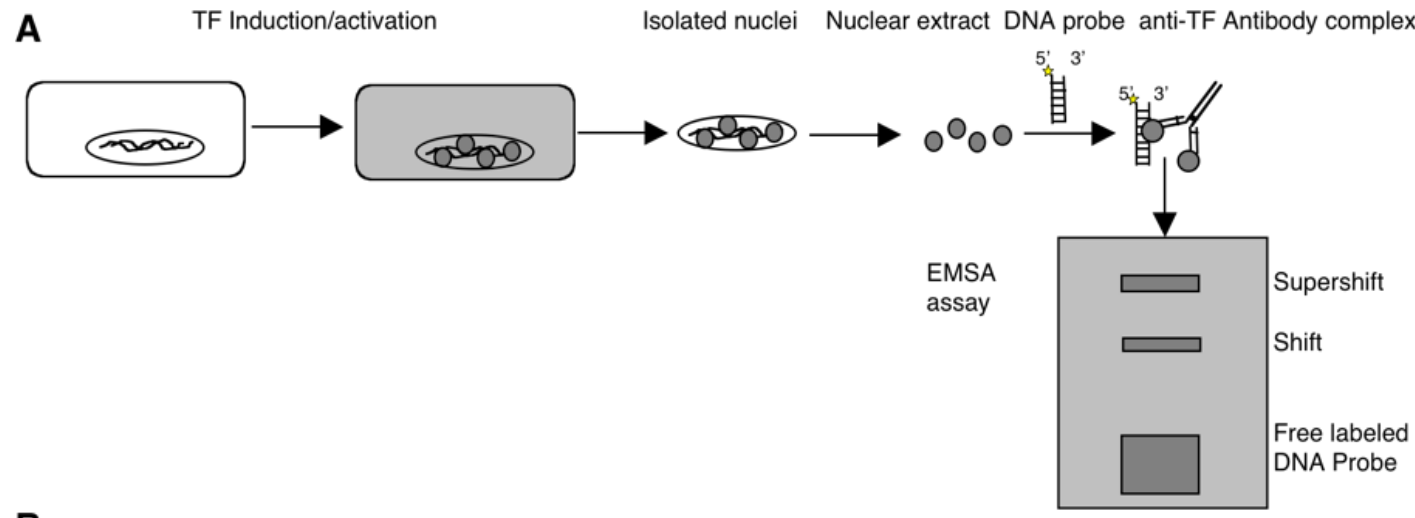

B

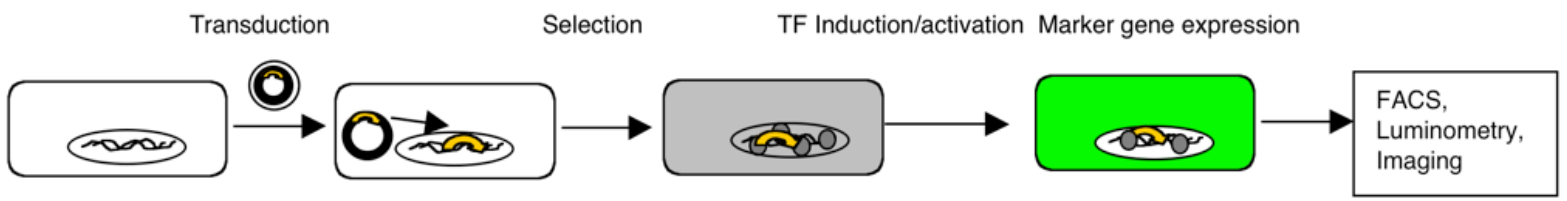

Figure 1. The major existing transcription factor functional analysis techniques : A) electrophoretic mobility shift assay EMSA (ex vivo, cell lysate based); B) marker protein expression cassette method (including knock-in models in vivo, cell line generation).

crosstalk, which is critically important for cell death and survival. We tested alternative technologies of TF activation detection in living cells and describe the results of ODN-MS testing in the model of early T1D that will be essential for further advancement of transcription activation imaging in vivo .

\subsection{Technologies of detecting transcription factor activation.}

The need to dissect gene transcription pathways and to elucidate the roles of individual protein components in transcription of any gene of interest necessitated developing TF analysis techniques. The armamentarium of molecular biology includes commonly used in situ and in vitro methods of transcription factor analysis (Fig 1). In situ approach requires cell extraction and/or tissue sampling, isolation of cell nuclei followed by either subsequent analysis of total protein of interest (electrophoretic separation and detection) or, alternatively the analysis of functionally active factor - by electrophoretic mobility shift analysis in the presence of labeled «target» DNA sequences (Fig. 1A). As a result, the observed shift is a consequence of large protein-DNA complex's slower migration in electric field in the presence of the polyacrylamide gel meshwork. The latter migration shift can be made more prominent by increasing the mass of the complex with antibodies that specifically bind to transcription factor-DNA probe complex and retard the migration even further. Similar approach can be adapted to ELISA-like format with TF-binding probes linked to the surface of the plate that does not require electrophoretic separation for detecting TF-probe interaction. The need of non-destructive methods of gene transcription detection in live cells prompted the introduction of reporter gene constructs (DNA vectors, Fig. 1B). These vectors carry a copy of a reporter gene (luciferase, green fluorescent protein etc.) positioned under the control of a minimal promoter and regulatory element consisting of one or more TF binding elements, i.e. DNA sequences that specifically bind transcription factors initiating gene transcription. 
The resultant gene expression cassette reports on the synthesis of downstream gene products (mRNA or protein) that can be easily detected either in cell extracts, whole live cells or even in live transgenic animals carrying expression cassettes with multiple NF-kB response elements regulating a minimal promoter and firefly luciferase cDNA. The reporter constructs can be used to image LPS and TNF $\alpha$-triggered inflammation in various cells and whole animals $[14,15]$. The genetic imaging reporters provide reproducible results if the same tissue types at similar anatomical locations are compared between animal groups. Some reporter proteins are expressed at quantifiable levels by using whole body imaging modalities (e.g. PET) that have few depth-related imaging limitations. Imaging of transcription regulation at the level of induction has been previously accomplished in vivo by using Herpes virus thymidine kinase (HSV1-tk)or dopamine receptor cDNAs positioned under the control of a tetracycline-inducible promoter. By using ${ }^{18} \mathrm{~F}$-labeled substrates and receptor ligands, correlative and doxycycline-inducible expression of both markers has been shown in animals harboring stably transfected C6 cells [16]. A p53dependent expression of HSV1-tk has been imaged in engineered tumors after treating animals with nitrosourea derivative which up-regulates p53. To impart sensitivity to p53 expression, an artificial promoter controlling HSV1tk -GFP fusion has been constructed. The promoter contained artificial regulatory element composed of a multiple tandem repeats of p53 binding sites [17]. Similar engineering approach and the same imaging marker gene were employed to image T-cell receptor dependent T-cell activation [18]. This has been achieved in a Jurkat lymphoma cell model using transcription activation via

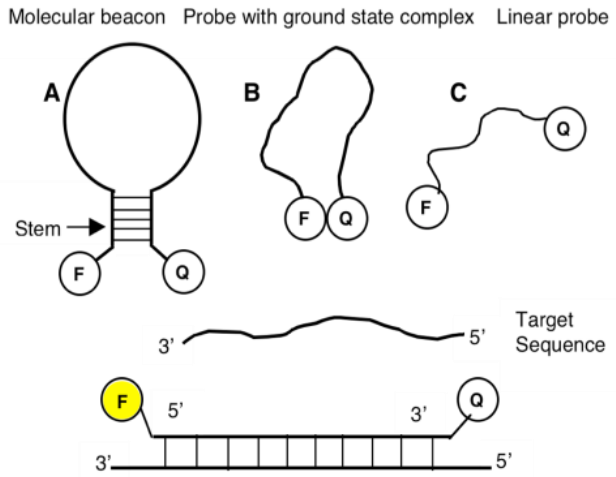

Figure 2. Structure of oligonucleotide beacons [6] nuclear factor (NFAT)- dependent mechanism. Cells were implanted in mice and showed a clear response to activation by intravenous administration of anti- Tcell receptor antibody. The response was imaged using both fluorescence of GFP and PET imaging with ${ }^{124}$ I-labeled FIAU substrate [18]. Vector-encoded marker expression cassettes are essential for successful engineering of "reporter cells" that could provide information about cytokine-mediated signaling. However, the above strategy does not allow straightforward comparative analyses of transcription factor activity or transcription factor concentration in various cells. Instead of imaging the event of active transcription factor binding to the DNA target motif, marker gene expression generates a protein product which is derivative of transcription/translation steps. This introduces the following variables: 1) marker gene mRNA stability in various cells; 2) protein folding rate; 3) proteolysis of the marker gene product. For example, a recent study

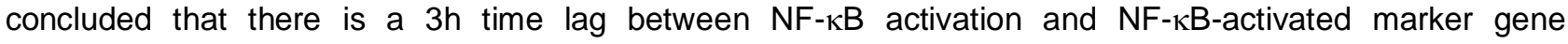
expression. In general, this suggests a significant spatial-temporal "disconnect" between TF activation and the "imageable" signal production [19]. Another limitation of marker gene technology is the requirement of reproducible cell transduction or transfection with a reporter construct, which is usually followed by selection of stable cell lines carrying the expression/reporter cassette. Therefore, the limited choice of in vivo TF detection strategies and the critical role of transcription regulators necessitates further research that would lead to non-invasive detection technologies and better quantitative evaluation of activated transcription factors of interest.

\subsection{Oligonucleotide (ODN)-based probes and beacons.}

Most of the imaging probes designed for detection of specific binding to TF were derived from short fragments of nucleic acids and fluorophores suitable for optical imaging. Optical imaging approach provides unparalleled flexibility of signal detection but has limitations due to the difficulty of signal quantitation in vivo. The direct detection of eukaryotic TF is a complex task since these proteins are recognizing double-helix elements and TF-DNA interaction does not result in strand separation that would simplify imaging signal encoding. Nevertheless, protein-duplex DNA interaction can be identified with optical methods, e.g. the effect of SYBR dye-labeled ODN duplex protection by bound proteins against degradation by exonucleases [20]. 
These SYBR ODN probes show high fluorescence only in the intact state (i.e. protected from the degradation by DNA-bound proteins). The detection of protected DNA is suitable mostly for in vitro analysis since SYBR can rapidly exchange with cellular DNA. The range of use of oligonucleotide-based probes (molecular beacons (MB) [21]) spans from in vitro detection of small molecules [22] to cell-based assays of complementary mRNAs [23]. Beacon technology is versatile, adaptable to a variety of analytical tasks due to variety of synthetic and half-synthetic approaches for

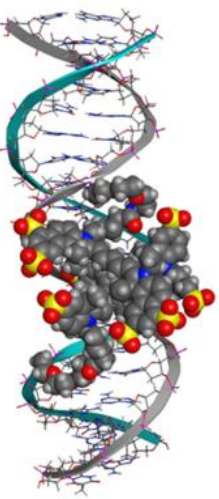

$0^{\circ}$
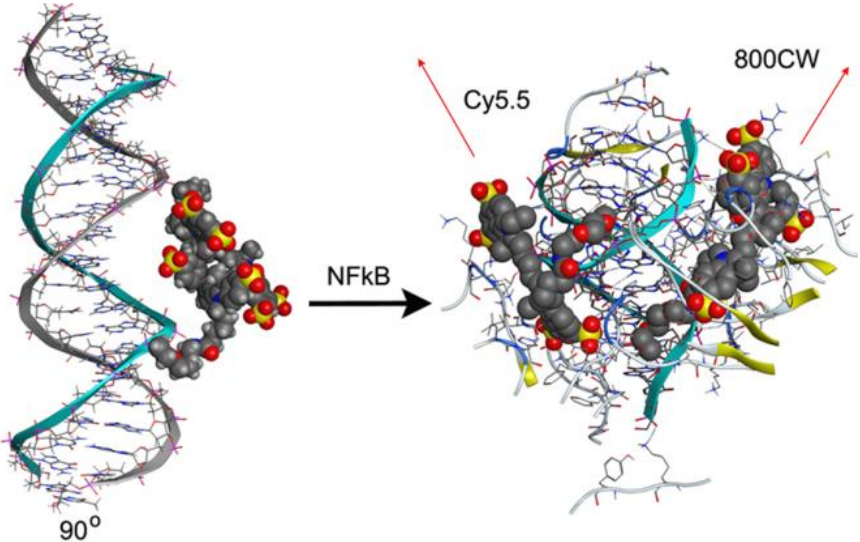

Figure 3. MOE-built model of ODN-MS with the NF-kB specificity carrying two NIR fluorophores, which form an interacting pair if positioned across the major groove of DNA. The pair has low fluorescence emission [7]. Upon binding with NF-kB there is a dissociation of the pair with resultant favorable orientation of transition dipole vectors (shown with arrows). Only those amino acids of NF- $\mathrm{BB}$ p50/p65 heterodimer interacting with ODN-MS (a fragment) and fluorophores were isolated and displayed. MOE molecular dynamics simulations were performed by using PDB1LE5 coordinates [9].

modification of primary and

secondary structure of oligonucleotide probes- from unique nucleotide substitutions with synthetic analogs [24] to aptamer evolution via molecular Selex technology [25]. The unique advantages of MB are: a) due to the presence of strong hydrogen-bond (Watson-Crick) interactions between nucleotide base pairs MB can store quenched (e.g. fluorescent or paramagnetic) molecular signals within a very small molecular frame; b) stability of above framework can be modified with single substitution of a single base pair; c) low molecular weight of $\mathrm{MB}$ and compact structure may facilitate easier intracellular uptake; d) various backbone modifications (e.g. phosphorothioate bonds, 2'-OMe, fluorination) enable to combat premature hydrolysis in living cells. The combination of these properties results in $\mathrm{MB}$ that are capable of high-specificity interaction with multiple analytes (mRNA, proteins and small molecules) which is followed by a detectable imaging signal due to destabilization (or stabilization) of MB structure, depending on the individual beacon design. Tagging oligonucleotides with imaging probes enables tracking these molecules in living cells and in vivo. For example, labeling of oligonucleotides with radioactive isotopes has been used for non-invasive biodistribution imaging. This approach potentially allows to localize the sites of target gene expression [26, 27]. Conjugation of oligonucleotides with NIR fluorophores and their quenchers could result in highly useful molecules with reporting capabilities. The latter is due to the effect of fluorescence quenching between the labeled proximal 3'-and 5' termini. The quenching effect is abrogated by de-quenching of fluorescence upon the interaction with in vitro targets in structurally constrained stem-loop beacons as well as stemless beacons (reviewed in $[6,28]$. In general, MBs were designed for the purpose of detecting complementary strands of mRNA (target sequence, Fig.2A): a stem-loop conformation initially pulls together 3 '- and 5 '- termini of a single DNA for positioning a fluorophore $(F)$ and a quencher $(Q)$ in a close proximity to each other (Fig. 2A) [29]. The stem structure coexists with a relatively large single-strand loop which encodes_complementarity to the target sequence. A short stem of this type of MB is required for the beacon to open seamlessly allowing the complementary sequence of MB to hybridize with the target mRNA (Fig. 2). The number of nucleotide bases in the loop should be properly «balanced» by the number of complementary base pairs in the stem due to the tendency of large loop-small stem beacons to lose poorly stabilized secondary structure. However, if $F$ and $Q$ strongly interact with each other, the stem element of $M B$ could be prevented from dissociating. In the latter case there is a chance of stemless beacon-linear probe (Fig. $2 \mathrm{~B}$ and $\mathrm{C}$ ) equilibrium shift towards the stabilization of stemless beacon structure. The chemical structure differences between various pairs of $F$ and $Q$ molecules in their ability to interact with each other allow fine tuning of quenching effects essential for molecular target identification. If fluorescence of $F$ spectrally overlaps with $Q$ 
absorbance a quenching of $\mathrm{F}$ may occur due to non-emissive energy transfer mechanism. In the case when both strands of MB stem are of the same length and are mostly complementary to each other (i.e. when Watson-Crick base pairing dominates) closely positioned $\mathrm{Q}$ and $\mathrm{F}$ can form a complex with static quenching properties, i.e. when light absorbance of two molecules no longer resemble a sum of two individual molecules but is comprised of entirely different heterodimer with unique spectral properties due to coupling of excited state energy levels. In general, quenchers and fluorophores form either $\mathrm{H}$ - (blue-shifted) or J-(redshifted) aggregates [6, 29]. Low or near-absent fluorescence in a ground-state of blue-shifted aggregates results in an extremely low background signal of the intact labeled MB. For the purpose of measuring in the range of the low intrinsic (background) absorbance various NIR dyes were explored as fluorochromes for MB labeling. The efficacy of quenching of these fluorophores may reach $\sim 97 \%$. The relative fluorescence increase upon dequenching is critical for MB efficacy as diagnostic imaging probes. More recent data suggests that alternative Selex technology-derived aptamers are also capable of specific binding to TF and they were adapted for imaging with delivery to cells facilitated by superparamagnetic nanoparticles [30].

\subsection{Oligonucleotide duplexes as transcription factor-directed drugs.}

Hairpin oligonucleotide duplexes (decoys) were initially designed to block the transcription of a target gene by competing with genomic DNA for relevant transcription factors [31]. In the past, TF decoys bearing a NF- $\kappa B$ recognition sequence which binds to dimers formed by $\mathrm{p} 50$ and p65 TF subunits, were modified with reactive nucleotide analogs that
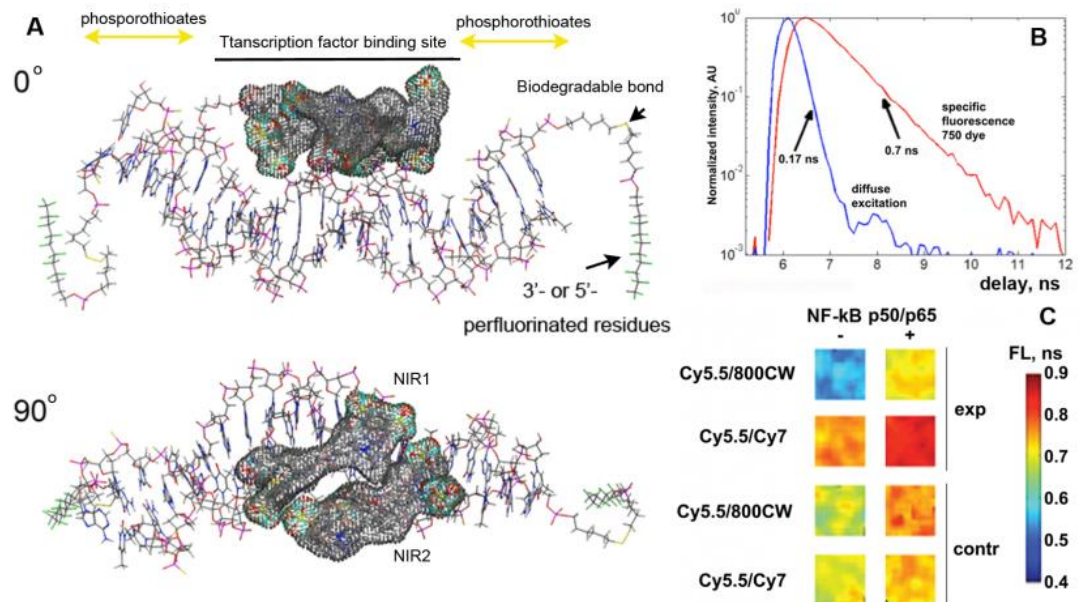

Figure 4. A- projection views of ODN-MS model at two angles of rotation. The sensor carries two interacting fluorophores [7] (in the major groove, rendered interacting surfaces are shown), and two short perfluorinated (PF) ODN end-modification tails [8]. B - the measurements of FL of a near-infrared fluorophore in the near-infrared range determined from mono-exponential decay curves can be easily separated from the short FL values of excitation pulse diffusely propagating through the tissue; $\mathbf{C}$ - pseudo-color FL maps of donor/acceptor pair linked to ODN-MS obtained in FRET mode ( $\lambda$ ex 650 $\mathrm{nm} / \lambda \mathrm{em} 800 \mathrm{~nm}$ ) showing the change of FL of the acceptor fluorophore after ODN-MS binding to TF. The maps were obtained by scanning solutions of ODN-MS probes bearing two interacting fluorophores (donor- Cy5.5 and acceptor $-800 \mathrm{CW}$ or Cy7, shown in $\mathrm{A}$ ) in the presence or in the absence of a mixture of purified NF- $\kappa B$ subunits. enabled covalent binding of TF and resultant "trapping" of NF-kB on the decoy and resulted in apoptosis of rapidly proliferating cells [32-34]. Oligonucleotide decoys were found efficient in inducing apoptosis in cancer cells [35] as well as in inhibiting neointima formation after balloon catheter induced arterial injury [36-38]. More recently TF ODN decoys with mono- and dual specificities were suggested for treating conditions caused by sustained inflammation [39, 40]. To assess the efficacy of intracellular penetration and to track their intracellular fate, decoys are labeled with molecular probes to make them detectable by imaging techniques, either microscopic, or macroscopic for in vivo imaging [41-44]. The major advantages of decoys as attenuators of gene expression are: 1) most of ODN decoys are doublestranded, compact and encode a protein-binding site without a need of unstable single-stranded loop; 2) in the case of rapidly activated TF decoys do not need to permeate into the nucleus as they «neutralize» TF in the cytoplasm either after translation and folding or immediately after the activation and dissociation from a complex with regulatory subunits (activity inhibitors). However, the major obstacle to a wide-scale use of decoys is the delivery across plasma membrane and tissue barriers to the sites of intended action [41]. The increased cellular tropism of oligonucleotide duplexes [45] and hairpins suggests that structure design and optimization of decoys can potentially greatly increase their biological activity. 


\section{RESULTS AND DISCUSSION}

\subsection{Oligonucleotide duplex probes for imaging binding to transcription factors.}

ODN-MS were initially designed and synthesized for detecting rapidly activated transcription factors (TF, such as NFKB $[4,5,46]$ and STAT3 [8]). The detection of these TF is feasible because ODN-MS does not have to penetrate into the cell nucleus to reach active transcription factors. Upon activation, the subunits of rapidly activated TF undergo release from inactive complexes with protein inhibitors (NF- $\mathrm{KB}$ family). Alternatively, they are activated by phosphorylation in the cytoplasm prior to transport through the nuclear membrane (STAT family). Activation in the cytoplasm provides a high concentration of binding centers for ODN-MS. Upon binding, the fluorophores linked to ODN-MS disengage from the non-fluorescent $(H-$ aggregates) they form within the major groove of the ODN duplex [7], then associate with interacting amino acid residues (e.g. arginine) within the TF binding site in such a way as to generate fluorescence. This fluorescence signal may be generated by the donor, the acceptor fluorophore, or change in FRET. The locations of fluorophore/amino acid interactions can be mapped using the available protein data base (PDB) data (Fig. 3). According to the results of simulations the close interaction between the donor and acceptor (or a quencher) with high likelihood is supposed to be strongly perturbed by DNAprotein interactions (Fig. 3). The ODN-MS transcription factor sensing, and detection mechanisms also provide an opportunity to use fluorescence lifetime (FL) changes for imaging rather than fluorescence intensity changes. FL has important advantages over the constant wavelength excitation because FL readout signal does not depend on local dye concentration, and the measured FL signal is independent of fluorescence excitation light intensity. FL measurements are also very sensitive to ODN-MS interactions with TF targets because the FL change reflects differences in fluorophore microenvironment before and after the binding event, e.g. time-domain imaging (FL-TD) resolves lifetimes corresponding to free and bound forms of ODN-MS [46]. As has been shown by performing fluorescence lifetime microscopy and by performing $F L$ of isolated organs, changes in microenvironment are ultimately quantifiable since FL decay curves can be subjected to multiexponential analysis to discriminate between transcription factor-bound vs. free components. The imaging setup previously developed by us is currently
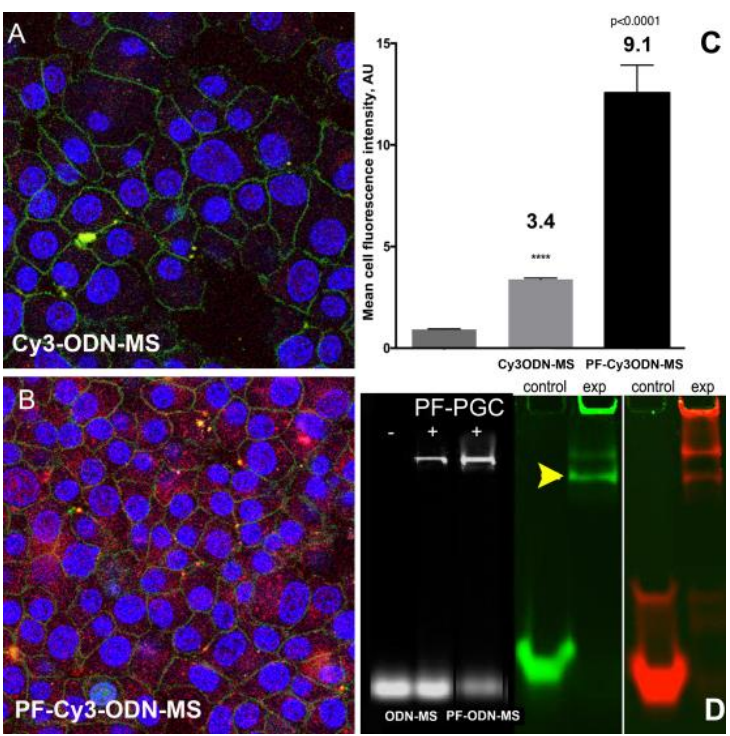

Figure 5. Red fluorescent Cy3-ODN-MS shows low levels of permeation into INS-1 cells $(\mathbf{A})$, the permeation into INS-1 improves 3-times over ODNMS levels by linking a single PF residue (B); (C)quantitative analyses of cell fluorescence intensity). D - PF-ODN-MS labeled with $800 \mathrm{CW}$ shows binding to fluorinated carrier polymer F-PGC (three lanes on the left) with improved specificity to STAT3 binding (shown in green, arrowhead) if compared to commercially available NIR STAT3 probe (red). used for performing multiexponential-analysis for deconvoluting discrete fluorescence lifetimes [47]. Multiexponential analysis along with the use of a prototype of time domain diffuse fluorescence tomography system [48] potentially allows robust recovery of the lifetime components present within the imaging volume in the case of for whole body imaging in small animals. To perform optimization of ODN-MS structure that would improve both detection of TF targets and intracellular delivery we applied in silico modeling together with synthetic approaches using a novel combination of internucleoside- and ODN end-linkers. Since successful delivery of ODN-MS probes will likely require protecting short oligonucleotide duplexes from rapid removal and degradation we considered various probe/carrier molecule interacting environments. We implemented two chemical strategies for improving ODN-MS performance: a) addition of a fluorophore pair to reporting on NF-KB (p50/p65) binding to ODN-MS using donor and acceptor FL; b) linking of short perfluorinated moieties to the termini of ODN-MS. We used molecular dynamics simulations of ODN-MS probes bearing two fluorophores (Fig. 4). These simulations were performed in molecular operating environment (MOE) to study potential formation of dye pairs within ODN-MS in which each ODN strand had a linked (donor or acceptor) NIR cyanine fluorophore. The results obtained by MOE modeling were in 
agreement with the actual spectroscopy performed on duplexes differing in the reciprocal orientation of the dye linking along the ODNs [7]. Two discrete cases were identified: 1) a Cy5.5/800CW dye pair located across the major groove interacting closely with strong overlap and suggested the formation of an $\mathrm{H}$-dimer as implied by the absorbance spectrum; and 2) Cy5.5/800CW dye pair across the minor groove of dsDNA favoring emissive FRET. Since the orientations of transition dipole moments of cyanines are known, MOE simulations provided reasonable prediction of emitting or nonemissive FRET and suggested that fluorophores positioned along the minor grove did not support the formation of a non-fluorescent dimers. The affinity of NF-kB for to the binding site defined by the sequence: 5'-GGGACTTTCC-3' (IgG kappa light chain promoter region) with a $\mathrm{Kd}$ in the range of single pM [49] suggesting high probability of TF/ODN-MS sensor complex formation even in the presence of fluorophore dimer close to the binding site. According to simulation results (Fig. 3), the non-emitting dimers formed across the major groove of the double helix may undergo dissociation. The measurements of FL of donor NIR fluorophore and acceptor in suggest that in both cases FL values increase in the presence of NF-kB heterodimers (p50/p65). We also applied ODN conjugation chemistry to test the modification of sensor's termini with short perfluorocarbons (PF). The increased uptake of the resultant PFODN-MS was anticipated due to membrane defects caused by hydrophobic/ lipophobic multifluorine- groups. Indeed, we observed a markedly improved uptake of PF-modified ODNMS in $\beta$-cell like INS-1 cells (Fig. 5). For imaging intracellular distribution, we used covalent internucleoside labeling of ODN-MS with Cy3 dye, which is resistant to bleaching. Images were obtained at a constant z-thickness and excitation so as to be directly comparable to the uptake of ODN-MS probes in 30-40 cells within 4-5 random fields of the FOV. Image intensity was quantified within ROls selected using cell perimeter delineation via fluorescent marker visualization of plasma membrane. ODN-MS carrying a single PF residue showed 3-times higher accumulation than control ODN-MS as determined by quantitative fluorescence microscopy of confocal optical sections in $\beta$-celllike INS-1 (Fig. 5) suggesting a significantly

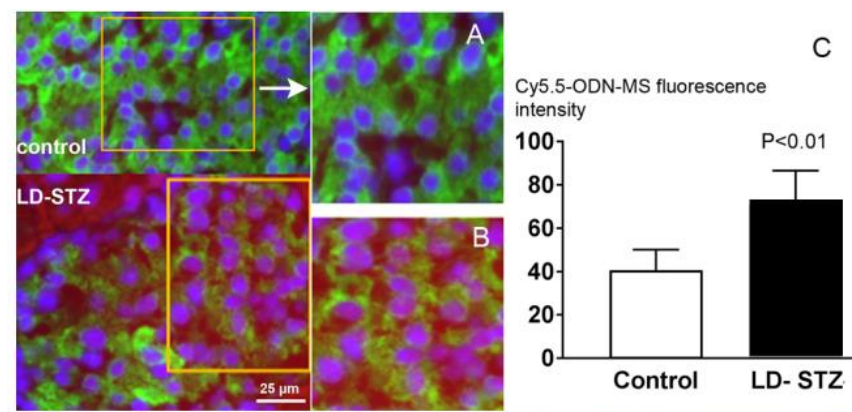

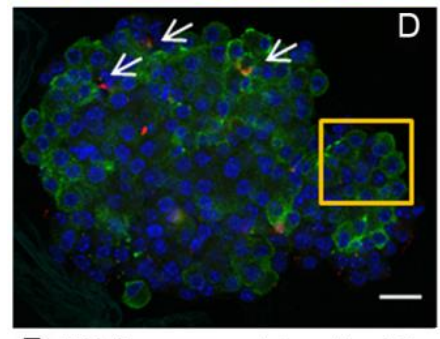

F NIR fluorecence intensity of the

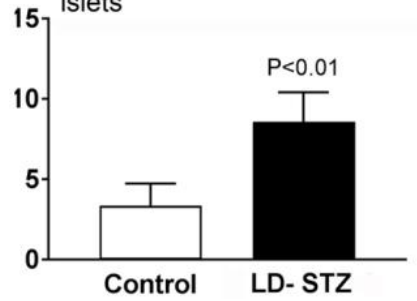
15 islets

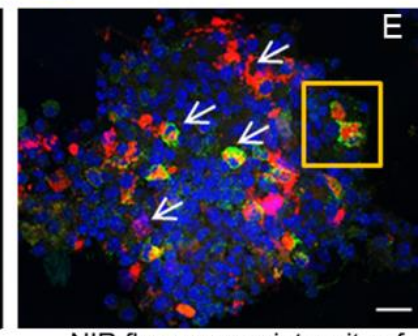

NIR fluorecence intensity of ${ }^{15}$

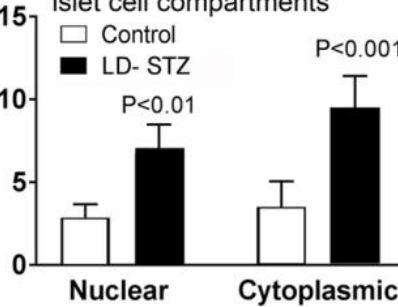

Figure 6. Microscopy and semi-quantitative assessment of Cy5.5-ODN-MS binding to NF-KB activation in pancreatic islets after low-dose streptozotocin (LD-STZ) treatment. ACy5.5-ODN-MS with NF-kB consensus sequence shows low levels of binding to the nuclei of cells in control islet sections (NF-kB (red); anti-insulin Ab (green), nuclei, (DAPI, blue); Bafter LD-STZ treatment both nuclear and cytoplasmic fluorescence of Cy5.5-ODN-MS was present in cells. Nuclear translocation of NF- $\mathrm{kB}$ is visible (magenta) after merging pseudo color red and blue channels. (250nM Cy5.5-ODN-MS in the presence of $\mathrm{Mg}^{2+}$, DTT, EDTA and tRNA following blocking with fragmented DNA in Denhardt's solution. 40x magnification; C- significantly higher $(p<0.01)$ cumulative fluorescence intensity of LD-STZ sections of pancreatic islets (closed bars) compared to control islets (open bars). D, EConfocal microscopic imaging of islets isolated from control (D) and LD-STZ treated (E) pancreas. Live islets were incubated with ODN-MS (300 nM), fixed and labeled with antiinsulin antibody. Scale bar $20 \mu \mathrm{m}$ ). LD-STZ treated islets contain insulin-positive cells with lower insulin content and a higher number of NIR positive cells compared to the control. Arrows point to islet cells (various insulin content) that were binding ODN-MS. F- quantification of ODN-MS fluorescence of live islets calculated using two confocal planes positioned 5 $\mu \mathrm{m}$ apart. LD-STZ treated islets show a higher signal compared to control islets. Fluorescence signal was higher $(P<0.01)$ in the nuclei and cytoplasm of the LD-STZ treated islets compared to control islets. 
higher cellular uptake in the case of PF-ODN-MS $(p<0.001)$. Higher Cy3 fluorescence intensity appeared to be associated with the nuclei of INS-1 cells both in the case of PF-modified or control ODN-MS. Our results demonstrate the feasibility of developing non-toxic modified ODN-MS probes for the purpose of delivering of imaging sensors into the cells. Conjugation of a single perfluorinated tail to ODN-MS was also resulting in improving the specificity of STAT3 recognition (as shown in Fig 5D) which is likely a consequence of a decrease in the levels of non-specific binding to other nuclear proteins.

\subsection{Imaging of TF activation in type 1 diabetes model}

Insulin-producing beta cells in the pancreas undergo continuous and extensive elimination months to years prior to the clinical onset of type 1 diabetes (T1D) due to autoimmune response. The detection of inflammation markers in pancreatic islets is essential for providing early diagnosis [50]. Early detection may enable therapeutic interventions that slow the progression of disease. Transcription factor NF- $\mathrm{B} B$ is at the center of regulating beta cell function while influencing the expression levels of multiple pro-inflammatory genes that affect the development of diabetes. Non-invasive imaging of NF-kB levels holds great promise for capturing the earliest inflammatory events in $\beta$-cells. Optical imaging techniques have a sensitivity advantage over alternative techniques that have a higher spatial resolution. For optical imaging in vivo and in situ, 20-50 times less imaging probe (in mass equivalents) is usually required compared to MR imaging. By employing sensing techniques with "smart/caged" fluorophore delivery capable of target protein sensing one could potentially reduce the required mass of imaging agent and improve target-to-background ratio due to the low imaging signal arising from non-target tissue compartments. The analysis of recent literature lends strong support to the theory that a balance between NF-kB and STAT TFs signaling is a key factor regulating $\beta$-cell death and survival and that any out of balance NF-KB/STAT activation in endocrine pancreas would point to a cell undergoing stress and diabetes progression. Thus, the ability to quantitatively assess the normal equilibrium concentration of both transcription factors represents a key metric in predicting the likelihood of disease development, and the ability to do so noninvasively without a biopsy would mark a major milestone in the early detection of diabetes. Therefore, we performed testing of previously designed and characterized ODN-MS in animal model of diabetes. We explored the use of sub-diabetogenic, low dose streptozotocin (LD-STZ) treatment that induces T1D through the activation of pro-inflammatory NF- $\kappa B$ signaling. LD-STZ treated SKH1 mice remained normoglycemic and microscopic imaging analysis showed that a significantly higher level of phosphorylated NF-kB-p65 expression $(\mathrm{P}<0.01)$ was present in the nuclei of islet cells compared to control animals. By using electrophoresis mobility shift assay (EMSA) we determined that the results of functional NF-kB detection were in agreement with the IF results: we observed a markedly higher specific FI of the NIR duplex probes in the islets of LD-STZ treated pancreas. Thus, we proved differential expression of NF-kB functional activity in LD-STZ treated tissues allowing distinguishing them from controls by a higher NIR intensity and a shift to longer lifetimes of NIR fluorophores. Optical sectioning of cells using confocal microscopy confirmed intracellular localization of NIR duplex in freshly isolated islets. Significantly higher NIR signal $(\mathrm{P}<0.01)$ of nuclei and cytoplasm of LD-STZ treated islet cells was in line with EMSA results and correlated with LD-STZ-induced activation of NF- $\mathrm{KB}$.

\section{CONCLUSIONS}

1) near-infrared (NIR) fluorescent probes consisting of a complementary pair of 21-base long oligonucleotides were synthesized by using previously designed transcription factor decoy molecules. These probes (ODN-MS) carried either a single NIR fluorophore (Cy 5.5) or a donor-acceptor NIR pair linked to ODN via internucleoside bonds; 2) MOE simulations and spectral measurements both suggest that if NIR fluorophores are linked across the major groove of a ODN duplex they form a low emitting, quenched dimer; 3) ODN-MS carrying such dimers and encoding as sequence specific for functional NF-kB heterodimer showed statistically significant increase of mean fluorescence lifetimes for both donor and acceptor (in FRET mode) after NF-kB heterodimer binding; 4) the experiments in T1D model using LD-STZ treatment schedule showed that ODN-MS has sufficient sensitivity to functional NF-kB in non-fixed islets and tissues; 5) ODNMS carrying donor NIR fluorophore only (i.e. Cy5.5) distinguishes between NF-kB expression in pre-diabetic vs. control pancreas by using FI and FLT techniques. These results are relevant for potential future in vivo 
translation NIR imaging of NF-kB activation in the pancreas and other organs affected by pro-inflammatory cytokine signaling due to local or systemic sustained inflammation.

Acknowledgment. This work was supported in part by NIH grants R01DK095728 and R01EB000858 (to A.B.). The authors are also grateful for the financial support for project No. 14.W03.31.0023 to the Ministry of Science and Higher Education of the Russian Federation.

\section{References}

[1] Ngo, V. N., Davis, R. E., Lamy, L. et al., "A loss-of-function RNA interference screen for molecular targets in cancer," Nature, 441(7089), 106-10 (2006).

[2] Imre, G., Gekeler, V., Leja, A. et al., "Histone deacetylase inhibitors suppress the inducibility of nuclear factor-kappaB by tumor necrosis factor-alpha receptor-1 down-regulation," Cancer Res, 66(10), 5409-18 (2006).

[3] Metelev, V., Weissleder, R., and Bogdanov, A., "Synthesis and properties of fluorescent NF-kappa B-recognizing hairpin oligodeoxyribonucleotide decoys," Bioconjugate Chemistry, 15(6), 1481-1487 (2004).

[4] Zhang, S., Metelev, V., Tabatadze, D. et al., "Near-infrared fluorescent oligodeoxyribonucleotide reporters for sensing NF-kappaB DNA interactions in vitro," Oligonucleotides, 18(3), 235-43 (2008).

[5] Zhang, S., Metelev, V., Tabatadze, D. et al., "Fluorescence resonance energy transfer in nearinfrared fluorescent oligonucleotide probes for detecting protein-DNA interactions," Proc Natl Acad Sci U S A, 105(11), 4156-61 (2008).

[6] Johansson, M. K., and Cook, R. M., "Intramolecular dimers: a new design strategy for fluorescencequenched probes," Chemistry, 9(15), 3466-71 (2003).

[7] Metelev, V., Zhang, S., Tabatadze, D. et al., "The three-dimensional context of a double helix determines the fluorescence of the internucleoside-tethered pair of fluorophores," Mol Biosyst, 9(10), 244753 (2013).

[8] Metelev, V., Zhang, S., Zheng, S. et al., "Fluorocarbons Enhance Intracellular Delivery of Short STAT3-sensors and Enable Specific Imaging," Theranostics, 7(13), 3354-3368 (2017).

[9] Berkowitz, B., Huang, D. B., Chen-Park, F. E. et al., "The x-ray crystal structure of the NF-kappa B p50.p65 heterodimer bound to the interferon beta -kappa B site," J Biol Chem, 277(27), 24694-700 (2002).

[10] Weissleder, R., Tung, C. H., Mahmood, U. et al., "In vivo imaging of tumors with protease-activated near-infrared fluorescent probes," Nature Biotechnology, 17(4), 375-378 (1999).

[11] Ntziachristos, V., Schellenberger, E. A., Ripoll, J. et al., "Visualization of antitumor treatment by means of fluorescence molecular tomography with an annexin V-Cy5.5 conjugate," Proceedings of the National Academy of Sciences of the United States of America, 101(33), 12294-12299 (2004).

[12] Metelev, V., Weissleder, R., and Bogdanov, A., Jr., "Synthesis and properties of fluorescent NFkappa B-recognizing hairpin oligodeoxyribonucleotide decoys," Bioconjugate Chemistry, 15(6), 1481-7 (2004).

[13] Zhang, S., Gupta, S., Fitzgerald, T. J. et al., "Dual radiosensitization and anti-STAT3 antiproliferative strategy based on delivery of gold nanoparticle - oligonucleotide nanoconstructs to head and neck cancer cells," Nanotheranostics, 2(1), 1-11 (2018).

[14] Ho, T. Y., Chen, Y. S., and Hsiang, C. Y., "Noninvasive nuclear factor-kappaB bioluminescence imaging for the assessment of host-biomaterial interac tion in transgenic mice," Biomaterials, 28(30), 4370-7 (2007).

[15] Robbins, D., and Zhao, Y., "Imaging NF-kappaB signaling in mice for screening anticancer drugs," Methods Mol Biol, 716, 169-77 (2011).

[16] Sun, X., Annala, A. J., Yaghoubi, S. S. et al., "Quantitative imaging of gene induction in living animals," Gene Therapy, 8(20), 1572-9 (2001).

[17] Doubrovin, M., Ponomarev, V., Beresten, T. et al., "Imaging transcriptional regulation of p53dependent genes with positron emission tomography in vivo," Proceedings of the National Academy of Sciences of the United States of America, 98(16), 9300-5 (2001). 
[18] Ponomarev, V., Doubrovin, M., Lyddane, C. et al., "Imaging TCR-dependent NFAT-mediated T-cell activation with positron emission tomography in vivo.," Neoplasia, 3(6), 480-488 (2001).

[19] Hellweg, C., Baumstark-Khan, C., and Horneck, G., "Generation of stably transfected Mammalian cell lines as fluorescent screening assay for NF-kappa B activation-dependent gene expression.," Journal of Biomolecular Screening, 8(5), 511-521 (2003).

[20] Chen, Z., Ji, M., Hou, P. et al., "Exo-Dye-based assay for rapid, inexpensive, and sensitive detection of DNA-binding proteins," Biochem Biophys Res Commun, 345(3), 1254-63 (2006).

[21] Tyagi, S., and Kramer, F., "Molecular beacons : probes that fluoresce upon hybridization.," Nature Biotechnology., 14(3), 303-308 (1996).

[22] Stojanovic, M. N., and Landry, D. W., "Aptamer-based colorimetric probe for cocaine," Journal of the American Chemical Society., 124(33), 9678-9 (2002).

[23] Marras, S. A., Kramer, F. R., and Tyagi, S., "Multiplex detection of single-nucleotide variations using molecular beacons," Genetic Analysis, 14(5-6), 151-6 (1999).

[24] Kurreck, J., "Antisense technologies. Improvement through novel chemical modifications. [Review] [131 refs]," European Journal of Biochemistry., 270(8), 1628-44 (2003).

[25] Rajendran, M., and Ellington, A. D., "In vitro selection of molecular beacons," Nucleic Acids Research., 31(19), 5700-13 (2003).

[26] MacLaren, D. C., Toyokuni, T., Cherry, S. R. et al., "PET imaging of transgene expression. [Review] [78 refs]," Biological Psychiatry, 48(5), 337-48 (2000).

[27] Younes, C. K., Boisgard, R., and Tavitian, B., "Labelled oligonucleotides as radiopharmaceuticals: pitfalls, problems and perspectives," Curr Pharm Des, 8(16), 1451-66 (2002).

[28] Broude, N. E., "Stem-loop oligonucleotides: a robust tool for molecular biology and biotechnology. [Review] [75 refs]," Trends in Biotechnology., 20(6), 249-56 (2002).

[29] Johansson, M., Fidder, H., Dick, D. et al., "Intramolecular Dimers: A New Strategy to Fluorescence

Quenching in Dual-Labeled Oligonucleotide Probes," J.Am. Chem.Soc., 124(24), 6950-6956 (2002).

[30] Liu, C. H., Ren, J., Liu, C. M. et al., "Intracellular gene transcription factor protein-guided MRI by DNA aptamers in vivo," FASEB J, 28(1), 464-73 (2014).

[31] Cho-Chung, Y. S., Park, Y. G., and Lee, Y. N., "Oligonucleotides as transcription factor decoys. [Review] [68 refs]," Current Opinion in Molecular Therapeutics, 1(3), 386-92 (1999).

[32] Metelev, V. G., Borisova, O. A., Volkov, E. M. et al., "New chemically reactive dsDNAs containing single internucleotide monophosphoryldithio links: reactivity of 5'-mercapto-oligodeoxyribonucleotides," Nucleic Acids Res, 29(19), $4062-9$ (2001).

[33] Metelev, V. G., Kubareva, E. A., Vorob'eva, O. V. et al., "Specific conjugation of DNA binding proteins to DNA templates through thiol-disulfide exchange," FEBS Lett, 538(1-3), 48-52 (2003).

[34] Lesage, D., Metelev, V., Borisova, O. et al., "Specific covalent binding of a NF-kappaB decoy hairpin oligonucleotide targeted to the p50 subunit and induction of apoptosis," FEBS Letters, 547(1-3), 115-8 (2003).

[35] Park, B. J., Brown, C. K., Hu, Y. et al., "Augmentation of melanoma-specific gene expression using a tandem melanocyte-specific enhancer results in increased cytotoxicity of the purine nucleoside phosphorylase gene in melanoma," Human Gene Therapy, 10(6), 889-98 (1999).

[36] Kume, M., Komori, K., Matsumoto, T. et al., "Administration of a decoy against the activator protein-1 binding site suppresses neointimal thickening in rabbit balloon-injured arteries," Circulation, 105(10), 1226-32 (2002).

[37] Nakamura, T., Morishita, R., Asai, T. et al., "Molecular strategy using cis-element 'decoy' of E2F binding site inhibits neointimal formation in porcine balloon-injured coronary artery model," Gene Therapy, 9(8), 488-94 (2002).

[38] Buchwald, A. B., Wagner, A. H., Webel, C. et al., "Decoy oligodeoxynucleotide against activator protein-1 reduces neointimal proliferation after coronary angioplasty in hypercholesterolemic minipigs," Journal of the American College of Cardiology, 39(4), 732-8 (2002).

[39] Hecker, M., and Wagner, A. H., "Transcription factor decoy technology: A therapeutic update," Biochem Pharmacol, 144, 29-34 (2017). 
[40] Miyake, T., Miyake, T., Sakaguchi, M. et al., "Prevention of Asthma Exacerbation in a Mouse Model by Simultaneous Inhibition of NF-kappaB and STAT6 Activation Using a Chimeric Decoy Strategy," Mol Ther Nucleic Acids, 10, 159-169 (2018).

[41] Thierry, A. R., Vives, E., Richard, J. P. et al., "Cellular uptake and intracellular fate of antisense oligonucleotides," Curr Opin Mol Ther, 5(2), 133-8 (2003).

[42] Islam, A., Handley, S. L., Thompson, K. S. et al., "Studies on uptake, sub-cellular trafficking and efflux of antisense oligodeoxynucleotides in glioma cells using self-assembling cationic lipoplexes as delivery systems," Journal of Drug Targeting., 7(5), 373-82 (2000).

[43] Hu, Q., Bally, M. B., and Madden, T. D., "Subcellular trafficking of antisense oligonucleotides and down-regulation of bcl-2 gene expression in human melanoma cells using a fusogenic liposome delivery system," Nucleic Acids Research., 30(16), 3632-41 (2002).

[44] Marcusson, E. G., Bhat, B., Manoharan, M. et al., "Phosphorothioate oligodeoxyribonucleotides dissociate from cationic lipids before entering the nucleus," Nucleic Acids Research., 26(8), 2016-23 (1998).

[45] Astriab-Fisher, A., Fisher, M. H., Juliano, R. et al., "Increased uptake of antisense oligonucleotides by delivery as double stranded complexes," Biochem Pharmacol, 68(3), 403-7 (2004).

[46] Bogdanov, A. A., Jr., Metelev, V., Zhang, S. et al., "Sensing of transcription factor binding via cyanine dye pair fluorescence lifetime changes," Mol Biosyst, 8(8), 2166-73 (2012).

[47] Goergen, C., Chen, H., Bogdanov, A. J. et al., "In vivo fluorescence lifetime detection of an activatable probe in infarcted myocardium," Journal of Bomedical Optics, 17(5), DOI: 10.1117/1.JBO.17.5.056001 (2012).

[48] Kumar, A. T., Raymond, S. B., Dunn, A. K. et al., "A time domain fluorescence tomography system for small animal imaging," IEEE Trans Med Imaging, 27(8), 1152-63 (2008).

[49] Zabel, U., Schreck, R., and Baeuerle, P. A., "DNA binding of purified transcription factor NF-kappa B. Affinity, specificity, Zn2+ dependence, and differential half-site recognition," J Biol Chem, 266(1), 252-60 (1991).

[50] Mathis, D., Vence, L., and Benoist, C., "Beta-cell death during progression to diabetes," Nature, 414(6865), $792-8(2001)$. 